\title{
Global Crack Detection using Bispectral Analysis
}

\author{
Charles COURTNEY, Bruce DRINKWATER, Simon NEILD, Paul WILCOX, \\ Department of Mechanical Engineering, University of Bristol, Bristol, United Kingdom.
}

\begin{abstract}
A global non-destructive testing technique for detecting cracks in engineering parts has been developed. The technique uses the bispectrum to analyse the mixing of two ultrasonic sinusoidal waves in a sample. The bispectrum's insensitivity to noise allows more sensitive detection of mixing signals than the power spectrum. Two sinusoidal signals are generated by signal generators, amplified and applied to a piezo-ceramic disk bonded to a sample. The sample is excited at very-high-order modes of vibration. The response of the sample is measured by a second piezoceramic disk and the received signal analysed using the bispectrum signalprocessing technique. Frequency mixing occurs as a result of the non-linear behaviour of cracks in the structure. Experiments with fatigue-cracked steel beams demonstrate that fatigue cracks produce a strong mixing effect and that the bispectrum signal relating to the frequency mixing is sensitive to the length of the crack; allowing the quantification of the crack length. Progress is being made in applying the technique to more practical situations: the sensitivity of the technique to transducer position and the input signal parameters (frequency and amplitude) is quantified experimentally.
\end{abstract}

\section{Introduction}

This paper describes the use of ultrasonic acoustic modulation as a method of dectecting cracks in metal parts. The current work looks to extend our knowledge of the parameters affecting the technique first described by Hillis et al.[1]. The technique uses bispectral analysis to measure the mixing signal caused by the nonlinear behaviour of a sample excited with two continuous ultrasonic signals.

The nonlinear nature of crack-face contact behaviour can lead to the production of harmonics [2] and, where two frequencies are present, signals at the sum and difference frequencies [3, 4]. Attempts to use these effects to detect cracks have typically concentrated on using the power spectrum to quantify the signals produced by the nonlinear behaviour, although the advantages of the bispectrum in detecting quadratically-phase-coupled signals [5-9] has lead to some interest in using the bispectrum in crack detection [10-14]. As the increase in crack length results in an increase in the bispectrum value corresponding to the mixing frequency [1] that bispectrum value is useful as a measure of the damage.

\subsection{Experimental Setup}

A $400 \mathrm{~mm} \times 60 \mathrm{~mm} \times 60 \mathrm{~mm}$ mild steel beam is excited using a piezo-ceramic disk (diameter $15 \mathrm{~mm}$, thickness $2 \mathrm{~mm}$ ) bonded with cyanoacrylate to the sample and driven by a power amplifier connected to two function generators; each supplying a sinusoidal input so that the signal is the superposition of two sine waves. The signal is detected by a second piezo-ceramic disk (diameter $5 \mathrm{~mm}$, thickness $2 \mathrm{~mm}$ ) and recorded using a digital oscilloscope. Figure 1 shows the experimental setup. 
The samples were suspended using looped wires running under the samples approximately $5 \mathrm{~cm}$ from either end. Four samples were used: one undamaged and three with fatigue cracks of $5 \mathrm{~mm}, 15 \mathrm{~mm}$ and $25 \mathrm{~mm}$ respectively.

Frequencies in the range $100-500 \mathrm{kHz}$ were used, with the conditions that the higher frequency $\left(F_{2}\right)$ was not an integer multiple of the lower $\left(F_{1}\right)$ and the difference between the frequencies was greater than $100 \mathrm{kHz}$. In order to achieve sufficient signal strength each frequency was tuned to find local maxima in the output amplitude, corresponding to a stationary state of the system. The amplitude of each input signal was then adjusted to give equal output voltages at each driving frequency.

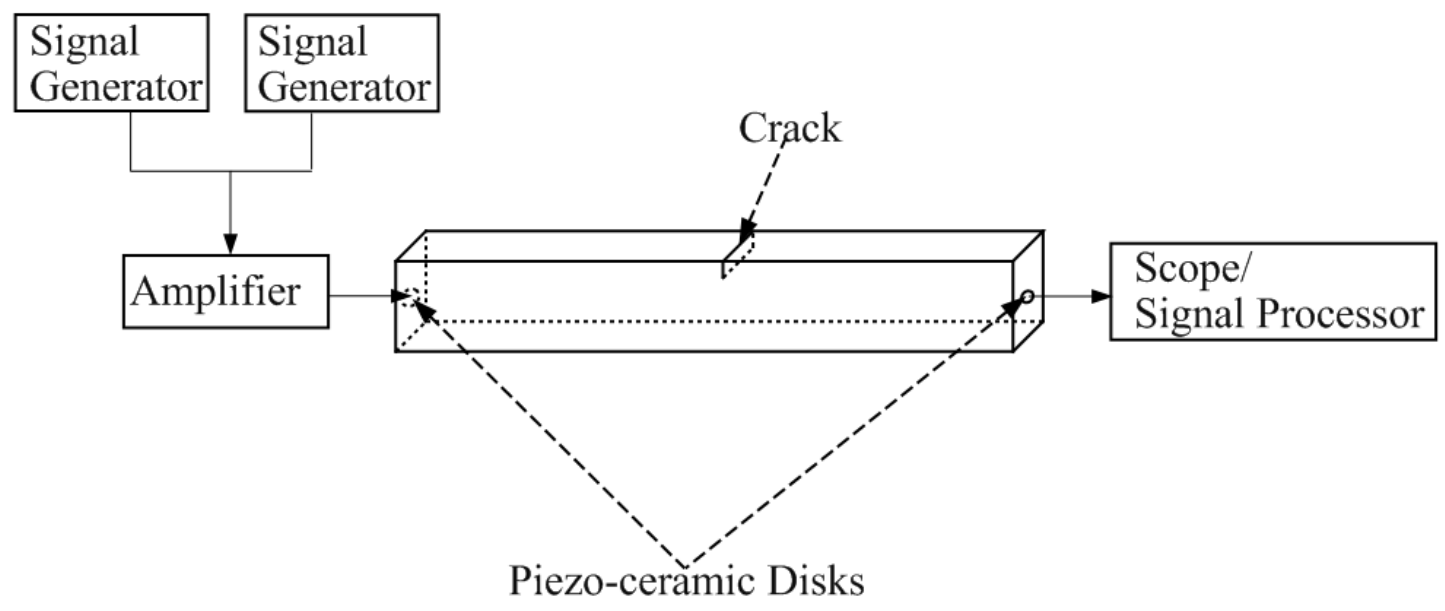

Figure 1: Experimental set-up.

\subsection{The Bispectrum}

Consider a time-domain signal, $S(t)$, with the Fourier transform $A(f)$. The power spectrum is

$P(f)=E\left[A(f) A^{*}(f)\right]$

and the bispectrum is

$B\left(f_{1}, f_{2}\right)=E\left[A\left(f_{1}\right) A\left(f_{2}\right) A^{*}\left(f_{1}+f_{2}\right)\right]$

where $E[]$ is the expectation value operator and $A^{*}(f)$ denotes the complex conjugate of the Fourier transform. In the current work the bispectrum is estimated directly by recording a long signal that is divided into $N$ shorter records labelled $n(=1,2, \ldots, N)$. The Fourier transform, $A_{n}(f)$, of each record is then calculated and the bispectrum is estimated as $A_{n}\left(f_{1}\right) A_{n}\left(f_{2}\right) A_{n}^{*}\left(f_{1}+f_{2}\right)$ averaged over the $N$ records $[12,13]$. bispectrum:

For a given pair of frequencies $f_{1}$ and $f_{2}$ there are four conditions for a non-zero

$$
\begin{aligned}
& A\left(f_{1}\right) \neq 0 \\
& A\left(f_{2}\right) \neq 0 \\
& A\left(f_{1}+f_{2}\right) \neq 0 \\
& \phi_{3}=\phi_{1}+\phi_{2}
\end{aligned}
$$

where $\phi_{1}$ and $\phi_{2}$ are the phases of the signals at $f_{1}$ and $f_{2}$ respectively and $\phi_{3}$ is the phase of the signal at $f_{1}+f_{2}$. 
500

450

(473kHz,473kHz)

400

350

300

(473kHz,269kHz)

$\stackrel{\text { N }}{\underset{\Sigma}{\Sigma}} 250$

200

150

100

50

$f_{1}=f_{2}$

0

100

$200 \mathrm{f}_{1}(\mathrm{kHz})$

400

500

Figure 2: Bispectrum resulting from exciting $60 \mathrm{~mm} \times 60 \mathrm{~mm} \times 400 \mathrm{~mm}$ steel sample at $269 \mathrm{kHz}$ and $473 \mathrm{kHz}$. A nonzero signal in the bispectrum (white indicates zero bispectrum in the plot) occurs where there is signal at three frequencies, $f_{1}, f_{2}$ and $f_{1}+f_{2}$. The peaks on the $f_{1}=f_{2}$ line result from second harmonic generation. The peaks at $269 \mathrm{kHz}, 204 \mathrm{kHz}$ and $473 \mathrm{kHz}, 269 \mathrm{kHz}$ result from signals at the sum and difference frequencies respectively. 


\section{Experimental Results}

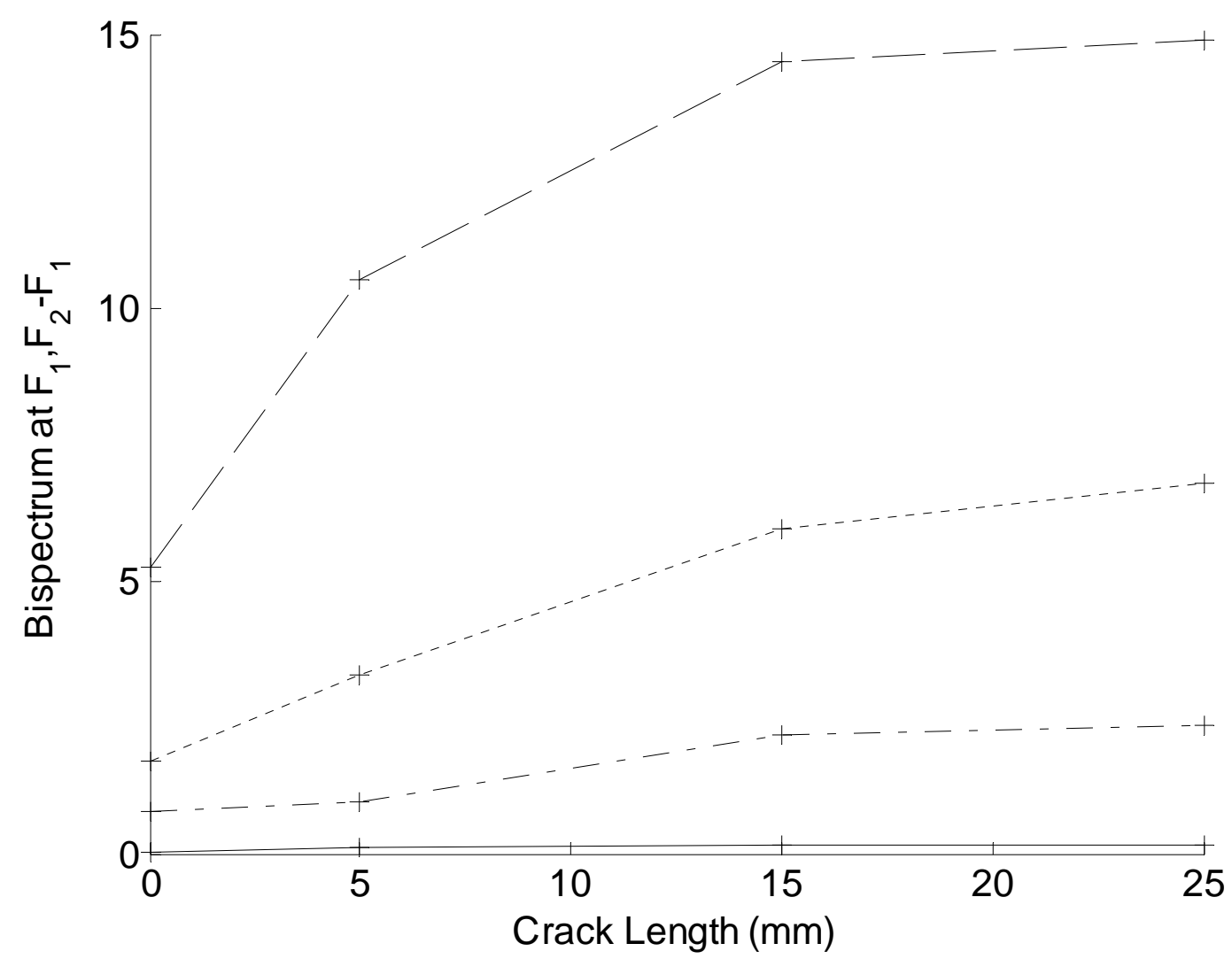

Figure 3: Bispectrum peak at $F_{1}, F_{2}-F_{1}$ from signal detected in steel beams with fatigue cracks of different lengths.

Samples were excited at $270 \mathrm{kHz}$ and $473 \mathrm{kHz}$ such that the output signal at each driving frequency is $1 \mathrm{~V}$ (solid line), $2 \mathrm{~V}(---), 3 \mathrm{~V}(--)$ and $4 \mathrm{~V}(--)$.

The samples were excited as described in section 1.1. The resulting bispectra took the form shown in figure 2 (note that some frequency smoothing has been performed for the sake of clarity), which shows the results for the $5 \mathrm{~mm}$-cracked sample excited at $269 \mathrm{kHz}$ and $473 \mathrm{kHz}$ with outputs at those frequencies of $1 \mathrm{~V}$ peak-to-peak. As the two frequencies are interchangeable the plot has reflection symmetry along $f_{1}=f_{2}$. The peaks on that line correspond to harmonic frequencies of the inputs combining with the input signals to conform with eqn. 3 . The off axis peaks correspond to the sum and difference frequencies. At no other point are the conditions of eqn. 3 met and so the bispectrum is zero at all other points. Hillis et al. [1] demonstrated that for a given set of parameters the value at $F_{1}, F_{2}-F_{1}$ is a useful measure of the sample damage: figure 3 shows that this is true across a range of vibration amplitudes (as measured by the voltage produced in the piezo-ceramic disk). The increase in the size of the peaks with increasing applied vibration amplitude closely follows the relationship $B\left(F_{1}, F_{2}-F_{1}\right) \propto V^{3}\left(F_{1}\right)$, where $V\left(F_{1}\right)=V\left(F_{2}\right)$ is the voltage to which the output signal amplitudes at $F_{1}$ and $F_{2}$ are set.

A number of factors affect the choice of applied frequencies. Experimental factors limit the range over which the current equipment allows generation and detection, without introducing significant nonlinearity, to $100 \mathrm{kHz}$ to $500 \mathrm{kHz}$. The frequencies at which it is possible to obtain a sufficient response are limited by the input and output transducer impulse response function and the modal behaviour of the sample itself. In order to test the validity of the technique at different frequencies a frequency region obeying all those conditions was required over which the frequency could be varied. With the lower 
frequency, $F_{1}$, held constant at $200 \mathrm{kHz}$, and adjusting the applied voltage such that the detected signal at $F_{1}$ and $F_{2}$ was $1 \mathrm{~V}$ peak-to-peak, the upper frequency, $F_{2}$, was increased from $290 \mathrm{kHz}$ to $370 \mathrm{kHz}$ in $10 \mathrm{kHz}$ increments. This process was repeated for the undamaged and least damaged (5mm fatigue cracked) samples and the resulting bispectrum amplitude at $F_{1}, F_{2}-F_{1}$ is show in figure 4 . The damaged sample consistently produced a larger signal than the undamaged, indicating that the technique is applicable over a range of frequencies. There is a variation in the degree of increase caused by the crack depending on the frequency, indicating that some frequency pairs will be more effective than others; however the increase at higher frequencies is due to approach of the mixing frequency $\left(F_{2}-\right.$ $F_{1}$ ) to $200 \mathrm{kHz}$ (the lower driving frequency) rather than an increase in the amount of mixing.

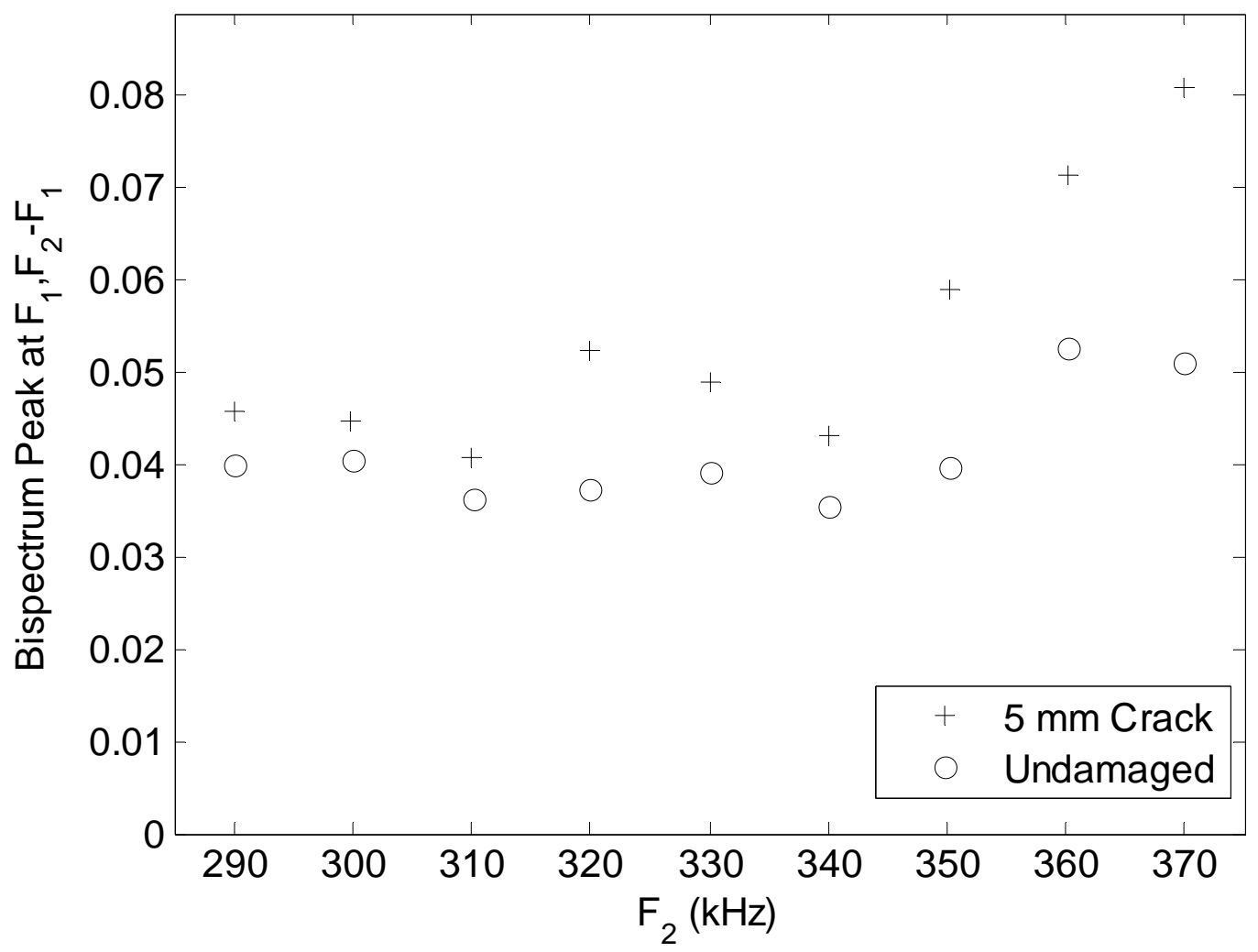

Figure 4: Variation of Bispectrum at $F_{1}, F_{2}-F_{1}$ with $F_{2}$ varied. $F_{1}=200 \mathrm{kHz}$ and the output voltage at each driving frequency was set to $1 \mathrm{~V}$ peak to peak.

The transducer positions shown in figure 1 were chosen on the assumption that they would excite and detect modes with strains perpendicular to the crack surfaces. This would be expected to produce a strong clapping non-linear behaviour. This hypothesis was tested by using transducers at two other positions, shown schematically in figure 5 alongside the resulting bispectrum peak values at $F_{1}, F_{2}-F_{1}$. Configuration A places both transducers on one side of the sample (we denote the smaller $60 \mathrm{~mm} \times 60 \mathrm{~mm}$ surfaces 'ends' and the $60 \mathrm{~mm} \times 400 \mathrm{~mm}$ surfaces 'sides') with the input transducer $40 \mathrm{~mm}$ from the end and the receiving transducer $50 \mathrm{~mm}$ from the input (90mm from the end). Configuration B places both transducers on one side of the sample with the input transducer $40 \mathrm{~mm}$ from the end and the receiving transducer $320 \mathrm{~mm}$ from the input. For comparison the results using the previous set-up are also shown, and the original, end-to-end configuration labelled configuration C. Each configuration was tested on all four samples with the peak-to-peak output amplitudes set to $1 \mathrm{~V}$. Configurations B and C were driven at $269 \mathrm{kHz}$ and $473 \mathrm{kHz}$, 
but for configuration $\mathrm{A}$ it was found that the response of the system was too small at $473 \mathrm{kHz}$ to produce viable results and so $269 \mathrm{kHz}$ and $440 \mathrm{kHz}$ were used.

Configuration A showed similar results to configuration $\mathrm{C}$ for the undamaged sample and the sample with the smallest crack, but higher values for both longer cracks. Configuration B behaved similarly to the original set-up (configuration C) at all crack lengths, but with larger bispectrum values and better contrast between crack lengths. For all three configurations there is a measurable increase in the peak as the crack length is increased.

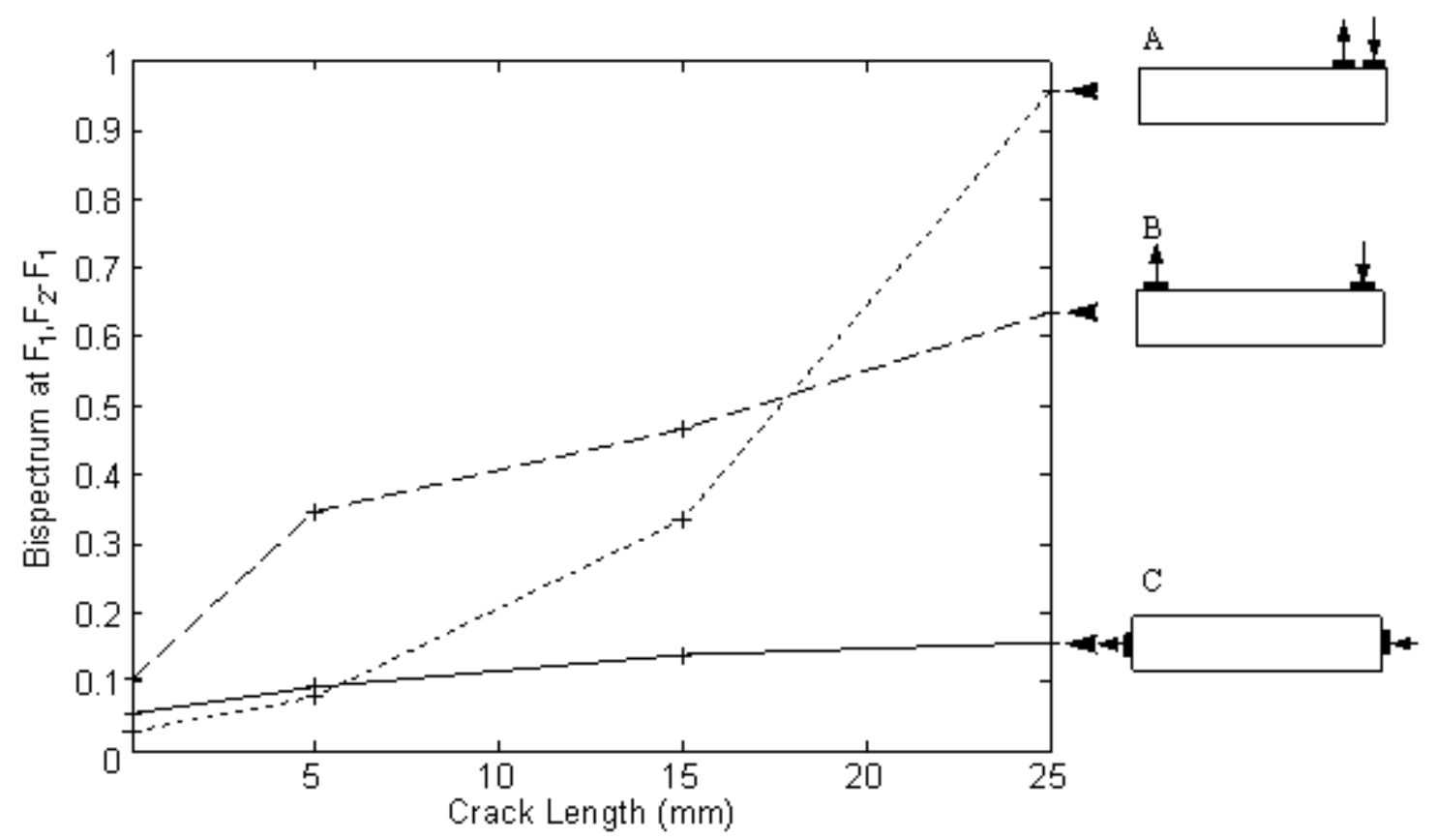

Figure 5: Variation of Bispectrum at $F_{1}, F_{2}-F_{1}$ with Increasing Crack Length for Three Different Transducer Set Ups. The three set ups were: Transducers positioned on ends of sample $(-)$, transducers $32 \mathrm{~cm}$ apart on the side of the sample $(--)$, both using $F_{1}=270 \mathrm{kHz}$ and $F_{2}=474 \mathrm{kHz}$, and transducers placed on the side, separated by $4 \mathrm{~cm}$ and excited using $F_{1}=270 \mathrm{kHz}$ and $F_{2}=440 \mathrm{kHz}(--)$.

The mixing signals are similar, or higher, for configurations $\mathrm{A}$ and $\mathrm{B}$, where the input transducer primarily oscillates parallel to the crack, than the original configuration, where the transducer operates perpendicular to the crack. This indicates that either the position of the transducer does not allow for straightforward selection of modes active in one direction, or the direction in which the stress acts does not affect the nonlinearity of the crack in the expected manner. Work is ongoing to measure the strain at the crack in order to determine the relationship between the strain at the crack and nonlinearity.

\section{Conclusions}

The bispectrum provides a useful tool to measure the mixing signal produced by nonlinearity in an ultrasonic experiment. The technique, exciting the sample with two ultrasonic frequencies and measuring the difference signal, proves consistent over a range of applied frequencies and vibrational amplitudes and works for a number of different excitation and detection positions. The different responses resulting from different transducer positions raises questions about how the vibrations interact with the crack, which will be addressed in our ongoing work to characterise better the behaviour, near the crack, of the vibrational modes excited, using modelling and strain measurements. 


\section{References}

[1] A. J. Hillis, S. A. Neild, B. W. Drinkwater and P. D. Wilcox. 2006."Global Crack Detection Using Bispectral Analysis," Proceedings of the Royal Society A, 462(2069):1515-1530

[2] I. Y. Solodov. 1998."Ultrasonics of Non-Linear Contacts: Propagation, Reflection and Nde-Applications," Ultrasonics, 36(1-5):383-390

[3] P. Duffour, M. Morbidini and P. Cawley. 2006."A Study of the Vibro-Acoustic Modulation Technique for the Detection of Cracks in Metals," Journal Of The Acoustical Society Of America, 119(3):1463-1475

[4] K. E. A. Van Den Abeele, A. Sutin, J. Carmeliet and P. A. Johnson. 2001."Micro-Damage Diagnostics Using Nonlinear Elastic Wave Spectroscopy (News)," NDT \& E International, 34(4):239-248

[5] W. B. Collis. 1998."Higher-Order Spectra: The Bispectrum and Trispectrum," Mechanical Systems and Signal Processing, 12(3):375

[6] J. W. A. Fackrell, P. R. White, J. K. Hammond and R. J. Pinnington. 1995."The Interpretation of the Bispectra of Vibrational Signals.1. Theory," Mechanical Systems and Signal Processing, 9(3):257-266

[7] J. W. A. Fackrell, P. R. White, J. K. Hammond, R. J. Pinnington and A. T. Parsons. 1995."The Interpretation of Bispectra of Vibration Signals.2. Experimental Results and Applications," Mechanical Systems and Signal Processing, 9(3):267-274

[8] C. L. Nikias. 1987."Bispectrum Estimation - a Digital Signal-Processing Framework," Proceedings of the IEEE, 75(7):869

[9] M. R. Raghuveer. 1985."Bispectrum Estimation - a Parametric Approach," IEEE Transactions on Acoustics, Speech, and Signal Processing, 33(5):1213

[10] J. Chen, I. Hagiwara, X. Su and Q. Z. Shi. 2002."A Bispectrum Feature Extraction Enhanced Structure Damage Detection Approach," JSME International Journal Series C-Mechanical Systems Machine Elements And Manufacturing, 45(1):121-126

[11] L. Gelman, P. White and J. Hammond. 2005."Fatigue Crack Diagnostics: A Comparison of the Use of the Complex Bicoherence and Its Magnitude," Mechanical Systems and Signal Processing, 19(4):913918

[12] P. J. Huber. 1971."Statistical Methods for Investigating Phase Relations in Stationary Stochastic Processes," IEEE Transactions on Audio and Electroacoustics, AU19(1):78

[13] K. S. Lii. 1981."Cross-Bispectrum Computation and Variance-Estimation," ACM Transactions on Mathematical Software, 7(3):284

[14] A. Rivola. 1998."Bispectral Analysis of the Bilinear Oscillator with Application to the Detection of Fatigue Cracks," Journal of Sound and Vibration, 216(5):889 